\title{
New Insights on the Mechanisms Affecting Fertility in Men with Non-Seminoma Testicular Cancer before Cancer Therapy
}

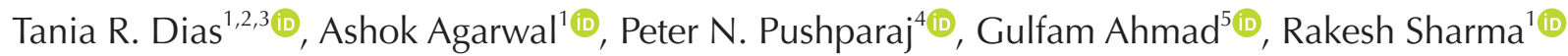 \\ ${ }^{1}$ American Center for Reproductive Medicine, Cleveland Clinic, Cleveland, OH, USA, ${ }^{2}$ Universidade da Beira Interior, Covilhã, Portugal, \\ ${ }^{3}$ Department of Microscopy, Laboratory of Cell Biology, Institute of Biomedical Sciences Abel Salazar and Unit for Multidisciplinary \\ Research in Biomedicine, University of Porto, Porto, Portugal, ${ }^{4}$ Center of Excellence in Genomic Medicine Research, Faculty of Applied \\ Medical Sciences, Jeddah, Saudi Arabia, ${ }^{5}$ Division of Pathology, School of Medical Sciences, Sydney University, Sydney, Australia
}

Purpose: Patients with non-seminoma testicular cancer (NSTC) cancer can be subfertile or infertile, and present reduced sperm quality, but the underlying mechanisms are unknown. The aim of this study was to compare the sperm proteome of patients with NSTC, who cryopreserved their sperm before starting cancer treatment, with that from healthy fertile men.

Materials and Methods: Semen volume, sperm motility and sperm concentration were evaluated before the cryopreservation of samples from patients with NSTC $(n=15)$ and the control group $(n=15)$. Sperm proteomic analysis was performed by liquid chromatography-tandem mass spectrometry and the differentially expressed proteins (DEPs) between the two groups were identified using bioinformatic tools.

Results: A total of 189 DEPs was identified in the dataset, from which five DEPs related to sperm function and fertilization were selected for validation by Western blot. We were able to validate the underexpression of the mitochondrial complex subunits NADH:Ubiquinone Oxidoreductase Core Subunit S1 (NDUFS1) and ubiquinol-cytochrome C reductase core protein 2 (UQCRC2), as well as the underexpression of the testis-specific sodium/potassium-transporting ATPase subunit alpha-4 (ATP1A4) in the NSTC group.

Conclusions: Our results indicate that sperm mitochondrial dysfunction may explain the observed decrease in sperm concentration, total sperm count and total motile count in NSTC patients. The identified DEPs may serve as potential biomarkers for the pathophysiology of subfertility/infertility in patients with NSTC. Our study also associates the reduced fertilizing ability of NSTC patients with the dysregulation of important sperm molecular mechanisms.

Keywords: Cancer therapy; Male fertility; Non-seminoma; Sperm quality; Testicular cancer

This is an Open Access article distributed under the terms of the Creative Commons Attribution Non-Commercial License (http://creativecommons.org/licenses/by-nc/4.0) which permits unrestricted non-commercial use, distribution, and reproduction in any medium, provided the original work is properly cited.

\section{INTRODUCTION}

Testicular cancer (TC) is a rare tumor among men, representing only $1 \%$ to $2 \%$ of all tumors [1]. The USA National Cancer Institute reported 8,850 new cases in 2017 [2] and this number is estimated to increase to

Received: Oct 23, 2018 Revised: Nov 22, 2018 Accepted: Nov 25, 2018 Published online Dec 21, 2018

Correspondence to: Ashok Agarwal (iD) https://orcid.org/0000-0003-0585-1026

Andrology Center, American Center for Reproductive Medicine, Cleveland Clinic, Mail Code X-11, 10681 Carnegie Avenue, Cleveland, OH 44195, USA.

Tel: +82-1-216-444-9485, Fax: +1-216-445-6049, E-mail: agarwaa@ccf.org 
9,310 new cases in 2018 [3]. The etiology of TC is not fully understood but several risk factors were identified, including genetic background, age, race/ethnicity, undescended testicle, human immunodeficiency virus infection and/or AIDS [4,5]. One of the main types of $\mathrm{TC}$ is non-seminoma testicular cancer (NSTC). It represents about half of the cases of germ cell tumors [6], which account up to $95 \%$ of all TC cases [7]. NSTC is commonly diagnosed in young men (25-35 years) [8,9], thus constituting a major threat for couples during reproductive years. Although the etiology of NSTC is largely unknown, its treatment is highly effective, showing a 5-year survival rate of $99 \%$ in early stages, and $48 \%$ to $91 \%$ in more advanced and metastatic stages [6]. Surgery, radiotherapy or chemotherapy are frequently used to treat this type of cancer [10]. However, these therapies are associated with secondary effects on male fertility, especially due to gonadal damage [11]. After the treatment, many patients show reduced sperm quality and impaired spermatogenesis, and often result in permanent infertility [12,13]. A 30\% decrease in the probability to conceive by natural conception after cancer therapy has been reported [14]. Thus, in most of NSTC cases the clinicians advise the patients to undergo sperm banking before initiating any kind of cancer therapy $[15,16]$. NSTC tends to grow rapidly and spread to other tissues [17], highlighting the urgency to initiate the treatment.

Most of the cases of NSTC are found when couples are not able to have children and seek for medical assistance. In fact, the development of NSTC is facilitated by the presence of abnormal semen parameters [18] and subfertility/infertility [19-21]. There are many cases of men presenting poor semen quality and reduced fertilizing ability prior to NSTC diagnosis [12,22,23]. Part of the problem starts with the impairment of spermatogenesis, as the tumor affects the normal hormonal regulation [24]. It is of extreme importance for the field of male reproduction to investigate the mechanisms by which NSTC affects male fertility. In this context, proteomics has emerged as a valuable tool to explore alterations in sperm proteome triggered by certain health conditions [25-27]. Due to their post-translational modifications, spermatozoa are highly susceptible to proteome alterations during maturation throughout the male reproductive tract [28]. These changes are highly dependent on the individual health status and may be a useful source of biomarkers to identify cer- tain disease conditions in its early stage. In this study, we aimed to analyze the sperm proteome of patients with NSTC who had cryopreserved semen samples before initiating cancer therapy and identify potential sperm protein biomarkers responsible for the altered reproductive function in these patients.

\section{MATERIALS AND METHODS}

\section{Study population and ethics statement}

This study was ethically approved by the Institutional Review Board (IRB) of Cleveland Clinic. The control group included 15 healthy volunteers with proven fertility, i.e., men who fathered a child in the last two years before entering the study, whereas the NSTC group comprised 15 NSTC patients who cryopreserved semen samples before starting cancer therapy. All the participants signed an informed written consent at the time of sample collection at the Andrology Center, Cleveland Clinic.

\section{Semen analysis and cryopreservation}

Semen samples were collected after 2 to 3 days of abstinence and placed at $37^{\circ} \mathrm{C}$ for 20 to 30 minutes to allow liquefaction. Volume, sperm motility, and sperm concentration were evaluated according to World Health Organization (WHO) 2010 guidelines [29]. Total sperm count and total motile count were also calculated and results were expressed as mean \pm standard error of mean (SEM). Semen samples were then cryopreserved in TEST-yolk buffer (TYB; Irvine Scientific, Santa Ana, CA, USA) in a ratio 1:1 as previously described [30] and finally stored in liquid nitrogen at $-196^{\circ} \mathrm{C}$.

\section{Sperm protein extraction and quantification}

Cryopreserved samples were thawed and centrifuged at $4,000 \times g$ for 10 minutes to isolate spermatozoa. The pellet was washed four times with consecutive resuspensions in phosphate buffered saline (PBS; Irvine Scientific) and centrifugations at 4,000×g for 10 minutes, at $4^{\circ} \mathrm{C}$. Radio-immunoprecipitation assay (RIPA; SigmaAldrich, St. Louis, MO, USA) buffer supplemented with Protease Inhibitor Cocktail, cOmplete ${ }^{\mathrm{TM}}$ ULTRA Tablets, ethylene diamine tetraacetic acid-free (Roche, Mannheim, Germany) was added to each sperm pellet (100 $\mu \mathrm{L}$ RIPA $/ 10^{6}$ sperm) and left overnight at $4^{\circ} \mathrm{C}$ for cell lysis. Then, samples were centrifuged at $10,000 \times g$ for 30 minutes, at $4^{\circ} \mathrm{C}$ and the supernatant containing 
the protein fraction was transferred to a new centrifuge tube. Protein quantification was performed using the Pierce BCA Protein Assay kit (Thermo Fisher Scientific, Waltham, MA, USA) according to the manufacturer's instructions.

\section{Liquid chromatography-tandem mass spectrometry}

The present study was conducted in compliance with the Minimum Information about a Proteomics Experiment (MIAPE) guidelines of the Human Proteome Organization's Proteomics Standards Initiative (HUPO-PSI) for reporting proteomics studies [31]. Three samples from control or NSTC groups ( $n=3 /$ group) were pooled using the same amount of protein from each sample and analyzed by liquid chromatographytandem mass spectrometry (LC-MS/MS). The samples in each pool were randomly selected within the experimental group and each pool was evaluated as an individual sample. Proteomic analysis was performed in a Finnigan LTQ-Obitrap Elite hybrid mass spectrometer using standard conditions and software [32,33]. The identification of the differentially expressed proteins (DEPs) between the control and NSTC groups was conducted via Scaffold (ver. 4.0.6.1; Proteome Software Inc., Portland, OR, USA) software. The abundance of each protein in the pool was classified as very low, low, medium or high based on the number of spectral counts. The normalized spectral abundance factor (NSAF) ratio was calculated to categorize the expression profile of DEPs as underexpressed, overexpressed or unique to one of the groups, as previously described [32].

\section{Ingenuity Pathway Analysis}

Bioinformatic analysis of the identified DEPs was performed through ingenuity pathway analysis (IPA; Qiagen, Hilden, Germany) software. This program allowed the evaluation of the top canonical pathways, diseases and bio functions, "Tox lists" and upstream regulators related to the DEPs. The criteria for the selection of DEPs for validation by Western blot (WB) included: 1) proteins involved in reproductive system development and function; 2) proteins involved in the top canonical pathways; 3) proteins with a higher difference of abundance between the experimental groups; 4) proteins with a well-described function in the literature. Proteins unique to the control group were not considered for protein validation.

\section{Validation of selected differentially expressed proteins}

Validation of key DEPs was performed by WB using six individual samples per group. Sperm proteins (25 $\mu \mathrm{g} / \mathrm{sample}$ ) were mixed with $4 \times$ Laemmli sample buffer in a ratio 1:3 in a total volume of $25 \mu \mathrm{L}$ in PBS. The prepared samples were immediately boiled at $95^{\circ} \mathrm{C}$ for 10 minutes for protein denaturation and ran into a $4 \%$ to $15 \%$ sodium dodecyl sulfate-polyacrylamide gel electrophoresis gel at constant voltage (90 V) for 2 hours. A molecular weight marker (Precision Plus Protein ${ }^{\text {TM }}$ Dual Xtra Standards) was loaded in the first well of each gel. Transfer of proteins from the gels to methanol-activated polyvinylidene difluoride membranes was performed with constant voltage (18 V) for 30 minutes. Membranes were blocked for 90 minutes at room temperature, with a $5 \%$ non-fat milk solution prepared in tris-buffered saline tween-20. Membranes were incubated overnight $\left(4^{\circ} \mathrm{C}\right)$ with specific primary antibodies followed by the respective secondary antibodies at room temperature, for 90 minutes (Supplemental Table 1). Images were acquired in a ChemiDoc ${ }^{\mathrm{TM}} \mathrm{MP}$ Imaging System after 5 minutes-incubation with enhanced chemiluminescence reagent (GE Healthcare, Marlborough, MA, USA) protected from the light. Image Lab ${ }^{\text {TM }}$ Software allowed quantification of bands density and the density of each band was divided by the corresponding total protein lane density. Results were expressed as fold change relative to the control group. All the reagents and equipment used for WB were purchased from BioRad (Hercules, CA, USA) unless specifically stated.

\section{Statistical analysis}

Semen parameters and WB results were analyzed by Mann-Whitney test for independent samples, using the MedCalc Software (ver. 17.8; MedCalc, Ostend,

Table 1. Semen parameters of fertile men (control) and patients with non-seminoma testicular cancer (NSTC)

\begin{tabular}{lccc}
\hline \multicolumn{1}{c}{ Parameter } & Control & NSTC & p-value \\
\hline Volume $(\mathrm{mL})$ & $3.53 \pm 0.35$ & $3.67 \pm 0.59$ & 0.7044 \\
Sperm motility $(\%)$ & $67 \pm 3$ & $59 \pm 7$ & 0.6936 \\
Sperm concentration $\left(10^{6} / \mathrm{mL}\right)$ & $95.49 \pm 7.79$ & $48.71 \pm 17.12$ & 0.0015 \\
Total sperm count $\left(10^{6}\right)$ & $316.92 \pm 45.41$ & $166.12 \pm 56.17$ & 0.0084 \\
Total motile count $\left(10^{6}\right)$ & $211.88 \pm 30.09$ & $108.36 \pm 35.95$ & 0.0136 \\
\hline
\end{tabular}

Values are presented as mean \pm standard error of mean $(n=15$ per group). Results were considered statistically significant for $\mathrm{p}<0.05$. 
Belgium). All data are presented as mean \pm SEM and differences with $\mathrm{p}<0.05$ were considered statistically significant.

\section{RESULTS}

\section{Semen parameters}

After assessing the semen parameters of fertile men (control) and patients with NSTC (Table 1), we observed that all the samples were within the normal standards of WHO [29]. The volume of ejaculate and sperm motility were similar between the groups, however there was a reduction in total motile count in NSTC group to about half of that in the control group ( $\mathrm{p}=0.0136$ ). Besides, sperm concentration and total sperm count were significantly lower $(p=0.0015$, and $\mathrm{p}=0.0084$, respectively) in NSTC patients in comparison to control group (Table 1).

\section{Identification of the differentially expressed proteins}

A total of 1,149 proteins were identified in the control group and 1,123 proteins in the NSTC group. Comparative proteomic analysis resulted in the quantification of 1,201 proteins, from which 189 were found to be DEPs between the experimental groups (Fig. 1). Most of the DEPs were underexpressed $(\mathrm{n}=105 ; 55.6 \%)$, while

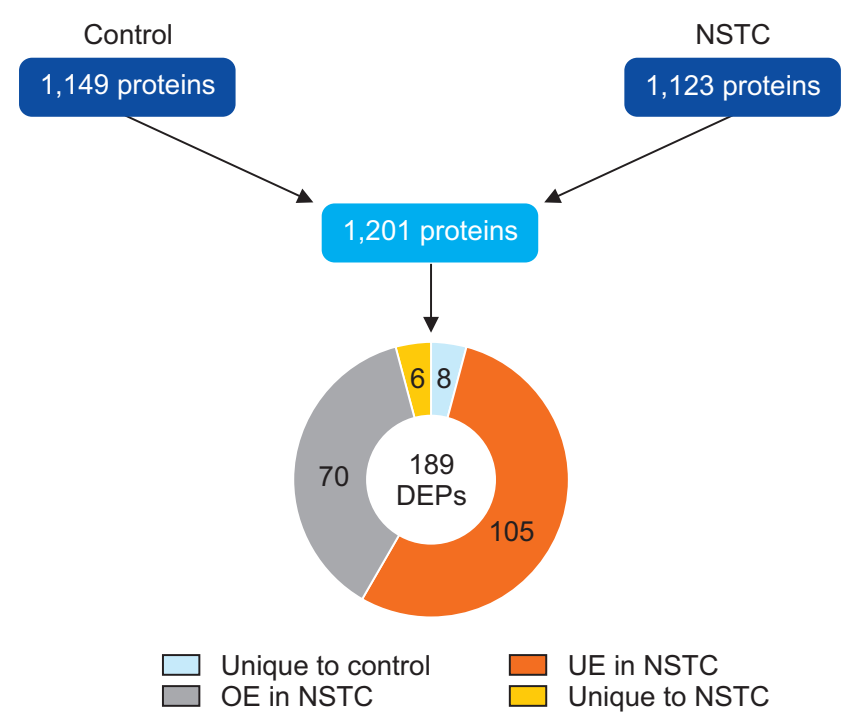

Fig. 1. Number of proteins identified by proteomic analysis of spermatozoa samples obtained from fertile men (control) and men with non-seminoma testicular cancer (NSTC), and expression profile of the differentially expressed proteins (DEPs) identified after comparative analysis between the experimental groups. UE: underexpressed, OE: overexpressed.
$37.0 \%(\mathrm{n}=70)$ were overexpressed in NSTC. Besides, $3.2 \%(n=6)$ were unique to NSTC group and $4.2 \%(n=8)$ unique to control group (Fig. 1).

\section{Selection of key differentially expressed protein for validation}

Through the IPA analysis, we observed that within the top 'canonical pathways' and 'Tox lists', many DEPs were involved in 'mitochondrial dysfunction'. Among those, we selected the proteins NADH-ubiquinone oxidoreductase $75 \mathrm{kDa}$ subunit (NDUFS1) and cytochrome b-c1 complex subunit 2 (UQCRC2), subunits of the mitochondrial complexes I and III, respectively. Besides, one of the top molecular and cellular functions in which the DEPs in this dataset were involved was 'free radical scavenging'. NDUFS1 together with annexin A2 (ANXA2) were included in this category, playing a role in the synthesis and metabolism of reactive oxygen species (ROS).

We also selected two proteins related to 'physiological system development and function': acrosin precursor (ACR) and sodium/potassium-transporting ATPase subunit alpha-4 (ATP1A4). The function of these proteins is important for specific reproductive processes (Table 2) intricate to fertilization. The abundance and expression pattern obtained by the proteomic analysis regarding the five selected proteins is presented in Table 3.

\section{Prediction of the upstream regulators}

The IPA software can predict the upstream regulators of the DEPs in a certain dataset. The activation/inhibition of certain regulatory proteins may explain the

Table 2. Specific functions of the differentially expressed proteins related to reproductive system development and function identified by the bioinformatic analysis when comparing the sperm proteome of patients with non-seminoma testicular cancer relative to fertile men

\begin{tabular}{llc}
\hline \multicolumn{1}{c}{ Process } & \multicolumn{1}{c}{ Protein } & p-value \\
\hline Cell movement of sperm & ATP1A4 & 0.0002 \\
Function of sperm & ATP1A4 & 0.0029 \\
Binding of sperm & ACR & 0.0041 \\
Function of reproductive system & ATP1A4 & 0.0100 \\
Fertilization & ACR, ATP1A4 & 0.0142 \\
Morphology of germ cells & ACR & 0.0165 \\
Fertility & ACR & 0.0231 \\
Penetration of zona pellucida & ACR & 0.0314 \\
\hline
\end{tabular}

ATP1A4: sodium/potassium-transporting ATPase subunit alpha-4, ACR: acrosin precursor. 
Table 3. Proteomic data of the differentially expressed proteins identified in the spermatozoa samples of fertile men (control) and men with nonseminoma testicular cancer (NSTC) before cancer therapy, which were selected for validation by Western blot

\begin{tabular}{|c|c|c|c|c|c|}
\hline \multirow{2}{*}{ Protein } & \multicolumn{2}{|c|}{ Abundance } & \multirow{2}{*}{ NSAF ratio } & \multirow{2}{*}{ Expression profile } & \multirow{2}{*}{$p$-value } \\
\hline & Control & NSTC & & & \\
\hline ACR & High & High & 0.60 & Underexpressed in NSTC & 0.0005 \\
\hline ANXA2 & Medium & High & 2.05 & Overexpressed in NSTC & 0.0003 \\
\hline ATP1A4 & Medium & Low & 0.47 & Underexpressed in NSTC & 0.0009 \\
\hline NDUFS1 & Medium & Very low & 0.40 & Underexpressed in NSTC & 0.0156 \\
\hline UQCRC2 & High & Medium & 0.61 & Underexpressed in NSTC & 0.0011 \\
\hline
\end{tabular}

NSAF: normalized spectral abundance factor, ACR: acrosin precursor, ANXA2: Annexin A2, ATP1A4: sodium/potassium-transporting ATPase subunit alpha-4, NDUFS1: NADH-ubiquinone oxidoreductase 75 kDa subunit, UQCRC2: cytochrome b-c1 complex subunit 2.

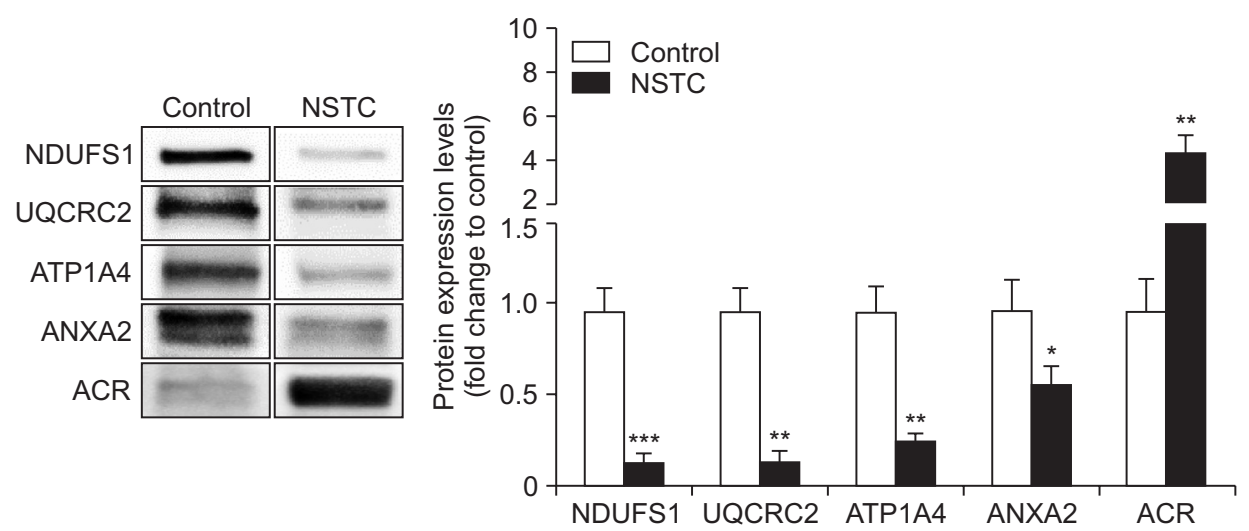

Fig. 2. Graphical representation of the expression levels of proteins involved in sperm function (NDUFS1, UQCRC2, ATP1A4, ANXA2, and ACR) in spermatozoa samples obtained from fertile men (control) and men with non-seminoma testicular cancer (NSTC). Results are presented as fold change to control and expressed as mean \pm standard error of mean ( $\mathrm{n}=6$ for each condition). Significantly different results relative to control are indicated as: * for $\mathrm{p}<0.05 ;{ }^{* *}$ for $\mathrm{p}<0.01 ;{ }^{* * *}$ for $\mathrm{p}<0.001$. Representative blots for each protein are also presented. NDUFS1: NADH-ubiquinone oxidoreductase $75 \mathrm{kDa}$ subunit, UQCRC2: cytochrome b-c1 complex subunit 2, ATP1A4: sodium/potassium-transporting ATPase subunit alpha-4, ANXA2: Annexin A2, ACR: acrosin precursor.

altered protein expression in the spermatozoa of NSTC group and provide information about the underlying mechanisms. The underexpression of the mitochondrial subunits NDUFS1 and UQCRC2 was predicted to be due to the activation of the rapamycin-insensitive companion of mammalian target of rapamycin (RICTOR; z-score 2.4). Moreover, the inhibition of the metalloendopeptidase OMA1 (OMA1; z-score -2.0) was also related to the underexpression of UQCRC2.

\section{Western blot validation}

We identifed the expression of all the selected DEPs through WB. We observed a significant decrease in the protein expression of NDUFS 1 from $1.00 \pm 0.13$ in the control group to $0.13 \pm 0.04$-fold change to control in the NSTC group (Fig. 2). A similar decrease was detected in the expression of UQCRC2 in NSTC group $(0.13 \pm 0.06$ fold change to control) relative to control $(1.00 \pm 0.14)$. WB results also showed a reduced expression of AT-
P1A4 and ANXA2 in patients with NSTC $(0.25 \pm 0.05$ and $0.57 \pm 0.11$-fold change to control, respectively) when compared to the control group $(1.00 \pm 0.14$ and $1.00 \pm 0.17$, respectively). On the other hand, there was a significant increase in the expression levels of ACR from $1.00 \pm 0.22$ in the control group to $5.95 \pm 1.06$-fold change to control in the NSTC group (Fig. 2).

\section{DISCUSSION}

Despite the significant advances in the field of male reproduction, the etiology of male subfertility/infertility is still poorly understood. About $15 \%$ to $30 \%$ of the infertility cases are diagnosed as idiopathic [34]. Patients with NSTC show similar reproductive disorders as those observed in infertile men [35]. It is important to investigate the molecular alterations at the spermatozoa level in patients with NSTC before initiating cancer therapy to understand the NSTC-related infer- 
tility and allow a premature non-invasive diagnosis. This is the first study focusing on the identification of sperm protein alterations in patients with NSTC before any treatment compared with healthy fertile men.

In spermatozoa, mitochondria are crucial for cellular energy production, survival and function [36]. Additionally, the mitochondrial respiratory chain is the main source of ROS. ROS play a key role in fertilization as they regulate sperm capacitation, hyperactivation, and acrosome reaction [37]. The mitochondrial complexes I and III are the principal sites of ROS generation [38]. They are constituted by several subunits, which are required for the functional assembly of the complexes [39]. For instance, NDUFS1 is the largest subunit of complex I, which by NADH oxidation provides electrons for the respiratory chain [40]. Besides, UQCRC2 is an essential component of complex III, another electron pump that contributes for oxidative phosphorylation (OXPHOS) [41]. Our proteomic data showed the underexpression of NDUFS1 and UQCRC2 in the NSTC group (Table 3), and these results were confirmed by the WB analysis (Fig. 2). The underexpression of NDUFS1 in the spermatozoa of NSTC men may lead to complex I dysfunction, which is one of the most common mitochondrial dysfunctions observed in humans [39]. Additionally, underexpression of UQCRC2 was associated with poor fertilization rates due to insufficient adenosine triphosphate (ATP) production and reduced sperm kinematics parameters [42]. Downregulation of NDUFS1 and UQCRC2 suggest mitochondrial dysfunction and subsequently abnormal ROS production that may result in oxidative stress. This has a major impact in sperm function, especially on the ability of spermatozoa to undergo capacitation and bind to the oocyte [43]. Oxidative stress is a common condition found in patients with NSTC and patients with other infertilityrelated disorders such as varicocele [44]. In fact, an underexpression of NDUFS1 and UQCRC2 was also observed in men with varicocele [44]. Furthermore, infertile varicocele patients with higher ROS production showed decreased sperm concentration and motility [45]. Thus, reduced total motile count, concentration and total sperm count observed in NSTC group (Table 1) may be explained by reduced ATP production and uncontrolled ROS production induced by mitochondrial dysfunction. Mitochondria are also susceptible to ROS attack, thus releasing pro-apoptotic molecules that will increase sperm cell death [46].
The underexpression of NDUFS1 and UQCRC2 was predicted to be due to activation of RICTOR, a component of the mammalian target of rapamycin (mTOR) pathway. This upstream regulator deserves special attention as the mTOR signaling cascade is responsible for the regulation of important cellular events, including cell growth and metabolism [47]. More specifically, RICTOR is a subunit of mTOR complex 2 (mTORC2), which is involved in cytoskeleton organization and insulin signaling $[48,49]$. The mechanisms by which mTOR regulates male fertility are not fully understood, but both complexes (mTORC1 and mTORC2) have been implicated in the regulation of blood-testisbarrier dynamics during spermatogenesis [50]. Though, a higher expression of RICTOR was observed in the early stages of the seminiferous epithelial cycle, while a progressive decline was observed at late stages [51]. Thus, activation of RICTOR in mature spermatozoa of NSTC patients may lead to a dysregulation of the mTOR system, affecting sperm quality. Inhibition or absence of mTOR has been reported to result in lower sperm production and decreased motility [50]. Our results indicate reduced sperm concentration, total sperm count and total motile count (Table 1) in NSTC group and these findings may be explained by an altered mTOR system in these patients. In fact, mTOR deregulation is common to many types of cancer [52] and should be explored as a mechanism of male fertility impairment in patients with NSTC. Furthermore, another upstream regulator, OMA1 was also associated with the underexpression of UQCRC2. OMA1 is a proteolytic enzyme located in the inner membrane of the mitochondria that is involved in the regulation of mitochondrial dynamics [53]. OMA1 deficiency severely affects the mitochondrial fusion-fission equilibrium, having important implications in cellular metabolic homeostasis [54]. Thus, the inhibition of OMA1 in spermatozoa of NSTC patients may result in mitochondrial dysfunction, further evidenced by the underexpression of not only UQCRC2, but also NDUFS1.

Among the identified DEPS, ATP1A4 was selected due to its participation in many reproductive processes, including sperm motility, function and fertilization, as well as function of reproductive system (Table 2). ATP1A4 is involved in the exchange of sodium and potassium ions across the plasma membrane, which are essential ions for sperm maturation [55]. Proteomic analysis demonstrated an underexpression of ATP1A4 
in NSTC group and this result was validated by the WB technique (Fig. 2). As ATP1A4 function is dependent on the availability of ATP [56], the downregulation of this protein is in accordance with the observed decrease in the expression of mitochondrial subunits NDUFS1 and UQCRC2, which consequently result in reduced ATP production. Previous studies reported that ATP1A4 plays a key role in human sperm motility $[57,58]$ and fertilizing ability in mice [55]. The underexpression of this protein may also be related to the reduction in total motile count seen in NSTC patients compared to fertile men (Table 1).

ANXA2 belongs to a family of calcium-dependent proteins that bind to negatively charged phospholipids in the cellular membrane [59]. This protein was identified in prostasomes, which are membrane-bound vesicles produced by the prostate epithelial cells [60] that are also present in the acrosome and tail of spermatozoa [61]. Prostasomes are rich in calcium and an increase in sperm intracellular calcium concentration was seen after fusion with prostasomes [62]. Calcium is very important for sperm motility acquisition and hyperactivation [63]. ANXA2 is believed to play a role in calcium transfer from prostasomes to spermatozoa, and is therefore important for sperm function [60]. Our proteomic analysis showed an overexpression of ANXA2 in NSTC group relative to control (Table 3). On the contrary, our WB results revealed a significant decrease in the expression levels of ANXA2 (Fig. 2) in patients with NSTC. We believe that our WB results are more reliable for the following reasons: i) use of a higher sample size for WB evaluation; ii) the intensity of the bands in each group (Fig. 2); and iii) the significant decrease in total motile count found in NSTC group (Table 1). In fact, a decreased mRNA expression of ANXA2 was associated with reduced motility [64]. In our study, the underexpression of ANXA2 in patients with NSTC suggests an insufficient calcium transfer from prostasomes, thereby contributing to the decrease in sperm quality.

The protein ACR was selected for validation due to its association with several reproductive processes, including binding of sperm, fertilization, morphology of germ cells, fertility, and penetration of zona pellucida (Table 2). The precursor form (inactive) of this protein proacrosin, was identified by the proteomic analysis as underexpressed in patients with NSTC. However, quantification of WB data showed a high overexpression of this protein in NSTC group (Fig. 2). Once again, we have contradicting results between the two techniques (proteomics vs. WB), but using a larger sample size for WB, we could observe by the representative bands (Fig. 2) that ACR is highly expressed in NSTC group. During acrosome reaction, proacrosin is converted to acrosin (active form) that plays a key role in sperm binding to the oocyte [65]. The overexpression of proacrosin in the spermatozoa of patients with NSTC may lead to an abnormally high production of acrosin, resulting in premature or defective acrosome reaction and impaired sperm-oocyte binding. Though, ANXA2 and ACR results need further confirmation with a larger sample size or an additional technique.

\section{CONCLUSIONS}

Altogether, our results indicate that patients with NSTC present an altered expression of proteins that are essential for sperm function and fertilization, especially those involved in sperm mitochondrial function (NDUFS1 and UQCRC2), motility (ATP1A4 and ANXA2), and fertilization (ATP1A2 and ACR). We validated the results obtained through the proteomic analysis by WB, showing the underexpression of NDUFS1, UQCRC2 and ATP1A4 in the spermatozoa of patients with NSTC relative to fertile men. Once these findings are reproduced by others in the field, we speculate that these three proteins may serve as potential biomarkers for the early and non-invasive clinical diagnosis of NSTC, as well as for new treatment approaches. In future, the analysis of the expression levels of these three proteins could be included into the routine evaluation of an infertile men as a screening for TC. Our data also highlight the importance of mTOR signaling pathway in the regulation of male fertility and that its possible dysregulation in spermatozoa of NSTC patients is related with reduced sperm quality and fertility potential. Although we found discordant results regarding the expression levels of ANXA2 and ACR, the alterations in these two proteins need further investigation in patients with NSTC as they may contribute to the understanding of the molecular basis of impaired fertility in these patients.

\section{ACKNOWLEDGEMENTS}

Financial support for this study was provided by the 
American Center for Reproductive Medicine, Cleveland Clinic, OH, USA. Tania R. Dias was supported by "Fundação para a Ciência e a Tecnologia" (FCT, SFRH/ BD/109284/2015) and Fulbright Program (E0585639). Sponsors were not involved in conducting the study or writing/submitting the paper.

The authors would like to thank to: Dr. Belinda Willard (Director, Proteomics Core Laboratory, Lerner Research Institute, Cleveland Clinic) for her support in the proteomic analysis; Dr. Ralf Henkel, Dr. Saradha Baskaran, and Dr. Manesh Kumar Panner Selvam for their help in reviewing the manuscript.

\section{Disclosure}

Authors have no potential conflicts of interest to disclose.

\section{Author Contribution}

Agarwal A and Sharma R designed the study and helped in data interpretation. Dias TR processed the samples, conducted the experiments and wrote the first draft of the manuscript. Pushparaj PN performed the bioinformatic analysis and Ahmad G helped in the collection/processing of samples. All authors read and approved the final manuscript.

\section{Supplementary Materials}

Supplementary materials can be found via https://doi. org/10.5534/wjmh.180099.

\section{REFERENCES}

1. Manecksha RP, Fitzpatrick JM. Epidemiology of testicular cancer. BJU Int 2009;104:1329-33.

2. Siegel RL, Miller KD, Jemal A. Cancer statistics, 2017. CA Cancer J Clin 2017;67:7-30.

3. Siegel RL, Miller KD, Jemal A. Cancer statistics, 2018. CA Cancer J Clin 2018;68:7-30.

4. Tvrda E, Agarwal A, Alkuhaimi N. Male reproductive cancers and infertility: a mutual relationship. Inter J Mol Sci 2015;16: 7230-60.

5. Garner MJ, Turner MC, Ghadirian P, Krewski D. Epidemiology of testicular cancer: an overview. Int J Cancer 2005;116: 331-9.

6. Oldenburg J, Fosså SD, Nuver J, Heidenreich A, Schmoll HJ, Bokemeyer C, et al. Testicular seminoma and non-seminoma: ESMO clinical practice guidelines for diagnosis, treatment and follow-up. Ann Oncol 2013;24 Suppl 6:vi125-32.

7. Eble JN, Sauter G, Epstein JI, Sesterhenn IA; World Health Organization, International Agency for Research on Cancer, International Academy of Pathology, editors. Pathology and genetics of tumours of the urinary system and male genital organs. Lyons: IARC Press; 2004;250-62.

8. Noone AM, Howlader N, Krapcho M, Miller D, Brest A, Yu M, et al. SEER cancer statistics review (CSR), 1975-2015. Bethesda: National Cancer Institute. 2009.

9. Oosterhuis JW, Looijenga LH. Testicular germ-cell tumours in a broader perspective. Nat Rev Cancer 2005;5:210-22.

10. Albers P, Albrecht W, Algaba F, Bokemeyer C, Cohn-Cedermark G, Horwich A, et al. Guidelines on testicular cancer. Eur Urol 2005;48:885-94.

11. Howell SJ, Shalet SM. Spermatogenesis after cancer treatment: damage and recovery. J Natl Cancer Inst Monogr 2005:12-7.

12. Ping P, Gu BH, Li P, Huang YR, Li Z. Fertility outcome of patients with testicular tumor: before and after treatment. Asian J Androl 2014;16:107-11.

13. Meistrich ML. Effects of chemotherapy and radiotherapy on spermatogenesis in humans. Fertil Steril 2013;100:1180-6.

14. Huyghe E, Matsuda T, Daudin M, Chevreau C, Bachaud JM, Plante $\mathrm{P}$, et al. Fertility after testicular cancer treatments: results of a large multicenter study. Cancer 2004;100:732-7.

15. Agarwal A. Semen banking in patients with cancer: 20 -year experience. Int J Androl 2000;23 Suppl 2:16-9.

16. Spermon JR, Kiemeney LA, Meuleman EJ, Ramos L, Wetzels AM, Witjes JA. Fertility in men with testicular germ cell tumors. Fertil Steril 2003;79 Suppl 3:1543-9.

17. Smith ZL, Werntz RP, Eggener SE. Testicular cancer: epidemiology, diagnosis, and management. Med Clin North Am 2018;102:251-64.

18. Jacobsen R, Bostofte E, Engholm G, Hansen J, Olsen JH, Skakkebaek NE, et al. Risk of testicular cancer in men with abnormal semen characteristics: cohort study. BMJ 2000;321: 789-92.

19. Eisenberg ML, Li S, Brooks JD, Cullen MR, Baker LC. Increased risk of cancer in infertile men: analysis of U.S. claims data. J Urol 2015;193:1596-601.

20. Walsh TJ, Croughan MS, Schembri M, Chan JM, Turek PJ. Increased risk of testicular germ cell cancer among infertile men. Arch Intern Med 2009;169:351-6.

21. Raman JD, Nobert CF, Goldstein M. Increased incidence of testicular cancer in men presenting with infertility and abnormal semen analysis. J Urol 2005;174:1819-22; discussion 1822.

22. Gandini L, Lombardo F, Salacone P, Paoli D, Anselmo AP, Culasso F, et al. Testicular cancer and Hodgkin's disease: 
evaluation of semen quality. Hum Reprod 2003;18:796-801.

23. Petersen PM, Skakkebaek NE, Vistisen K, Rørth M, Giwercman A. Semen quality and reproductive hormones before orchiectomy in men with testicular cancer. J Clin Oncol 1999; 17:941-7.

24. Morrish DW, Venner PM, Siy O, Barron G, Bhardwaj D, Outhet D. Mechanisms of endocrine dysfunction in patients with testicular cancer. J Natl Cancer Inst 1990;82:412-8.

25. Agarwal A, Bertolla RP, Samanta L. Sperm proteomics: potential impact on male infertility treatment. Expert Rev Proteomics 2016;13:285-96.

26. Sharma R, Agarwal A, Mohanty G, Du Plessis SS, Gopalan B, Willard B, et al. Proteomic analysis of seminal fluid from men exhibiting oxidative stress. Reprod Biol Endocrinol 2013;11: 85.

27. Intasqui P, Agarwal A, Sharma R, Samanta L, Bertolla RP. Towards the identification of reliable sperm biomarkers for male infertility: a sperm proteomic approach. Andrologia 2018;50.

28. Baker MA. Proteomics of post-translational modifications of mammalian spermatozoa. Cell Tissue Res 2016;363:279-87.

29. World Health Organization. WHO laboratory manual for the examination and processing of human semen. 5th ed. Geneva: World Health Organization; 2010.

30. Agarwal AS. Gupta S, Sharma R. Cryopreservation of client depositor semen. In: Agarwal AS. Gupta S, Sharma R, editors. Andrological evaluation of male infertility: a laboratory guide. Switzerland: Springer; 2016;113-33.

31. Martínez-Bartolomé S, Deutsch EW, Binz PA, Jones AR, Eisenacher M, Mayer G, et al. Guidelines for reporting quantitative mass spectrometry based experiments in proteomics. J Proteomics 2013;95:84-8.

32. Agarwal A, Ayaz A, Samanta L, Sharma R, Assidi M, Abuzenadah AM, et al. Comparative proteomic network signatures in seminal plasma of infertile men as a function of reactive oxygen species. Clin Proteomics 2015;12:23.

33. Agarwal AS. Gupta S, Sharma R. Reactive oxygen species (ROS) measurement. In: Agarwal AS. Gupta S, Sharma R, editors. Andrological evaluation of male infertility: a laboratory guide. Switzerland: Springer; 2016;155-63.

34. Practice Committee of the American Society for Reproductive Medicine. Effectiveness and treatment for unexplained infertility. Fertil Steril 2006;86:S111-4.

35. Agarwal A, Burns WR, Dada R, Sabanegh ES. Male infertility and testicular cancer-points of common causality. Euro Urol Rev 2010;5:56-9.

36. Ferramosca A, Zara V. Mitochondria and fertility: the mitochondria critical role on spermatozoa function. JDREAM 2017;1:21-6.
37. Aitken RJ. Reactive oxygen species as mediators of sperm capacitation and pathological damage. Mol Reprod Dev 2017; 84:1039-52.

38. Cassina A, Silveira P, Cantu L, Montes JM, Radi R, Sapiro R. Defective human sperm cells are associated with mitochondrial dysfunction and oxidant production. Biol Reprod 2015; 93:119.

39. Mimaki M, Wang X, McKenzie M, Thorburn DR, Ryan MT. Understanding mitochondrial complex I assembly in health and disease. Biochim Biophys Acta 2012;1817:851-62.

40. Janssen RJ, Nijtmans LG, van den Heuvel LP, Smeitink JA. Mitochondrial complex I: structure, function and pathology. J Inherit Metab Dis 2006;29:499-515.

41. Murphy MP. How mitochondria produce reactive oxygen species. Biochem J 2009;417;1-13.

42. Shukla KK, Kwon WS, Rahman MS, Park YJ, You YA, Pang MG. Nutlin-3a decreases male fertility via UQCRC2. PLoS One 2013;8:e76959.

43. de Lamirande E, O'Flaherty C. Sperm activation: role of reactive oxygen species and kinases. Biochim Biophys Acta 2008; 1784:106-15.

44. Samanta L, Agarwal A, Swain N, Sharma R, Gopalan B, Esteves SC, et al. Proteomic signatures of sperm mitochondria in varicocele: clinical utility as biomarkers of varicocele associated infertility. J Urol 2018;200:414-22.

45. Pasqualotto FF, Sharma RK, Nelson DR, Thomas AJ, Agarwal A. Relationship between oxidative stress, semen characteristics, and clinical diagnosis in men undergoing infertility investigation. Fertil Steril 2000;73:459-64.

46. Peña FJ, Rodríguez Martínez H, Tapia JA, Ortega Ferrusola C, González Fernández L, Macías García B. Mitochondria in mammalian sperm physiology and pathology: a review. Reprod Domest Anim 2009;44:345-9.

47. Saxton RA, Sabatini DM. mTOR signaling in growth, metabolism, and disease. Cell 2017;168:960-76.

48. Laplante M, Sabatini DM. mTOR signaling in growth control and disease. Cell 2012;149:274-93.

49. Dong H, Chen Z, Wang C, Xiong Z, Zhao W, Jia C, et al. Rictor regulates spermatogenesis by controlling sertoli cell cytoskeletal organization and cell polarity in the mouse testis. Endocrinology 2015;156:4244-56.

50. Oliveira PF, Cheng CY, Alves MG. Emerging role for Mammalian target of rapamycin in male fertility. Trends Endocrinol Metab 2017;28:165-7.

51. Mok KW, Mruk DD, Lee WM, Cheng CY. Rictor/mTORC2 regulates blood-testis barrier dynamics via its effects on gap junction communications and actin filament network. FASEB J 2013;27:1137-52. 
52. Xu K, Liu P, Wei W. mTOR signaling in tumorigenesis. Biochim Biophys Acta 2014;1846:638-54.

53. Baker MJ, Lampe PA, Stojanovski D, Korwitz A, Anand R, Tatsuta T, et al. Stress-induced OMA1 activation and autocatalytic turnover regulate OPA1-dependent mitochondrial dynamics. EMBO J 2014;33:578-93.

54. Quirós PM, Ramsay AJ, Sala D, Fernández-Vizarra E, Rodríguez F, Peinado JR, et al. Loss of mitochondrial protease OMA1 alters processing of the GTPase OPA1 and causes obesity and defective thermogenesis in mice. EMBO J 2012;31: 2117-33.

55. Jimenez T, McDermott JP, Sánchez G, Blanco G. Na,KATPase alpha4 isoform is essential for sperm fertility. Proc Natl Acad Sci U S A 2011;108:644-9.

56. Hlivko JT, Chakraborty S, Hlivko TJ, Sengupta A, James PF. The human Na,K-ATPase alpha4 isoform is a ouabain-sensitive alpha isoform that is expressed in sperm. Mol Reprod Dev 2006;73:101-15.

57. Koçak-Toker N, Aktan G, Aykaç-Toker G. The role of Na,KATPase in human sperm motility. Int J Androl 2002;25:180-5.

58. Sanchez G, Nguyen AN, Timmerberg B, Tash JS, Blanco G. The Na,K-ATPase alpha4 isoform from humans has distinct enzymatic properties and is important for sperm motility. Mol Hum Reprod 2006;12:565-76.
59. Bharadwaj A, Bydoun M, Holloway R, Waisman D. Annexin A2 heterotetramer: structure and function. Int J Mol Sci 2013; 14:6259-305.

60. Burden HP, Holmes CH, Persad R, Whittington K. Prostasomes: their effects on human male reproduction and fertility. Hum Reprod Update 2006;12:283-92.

61. Feinberg JM, Rainteau DP, Kaetzel MA, Dacheux JL, Dedman JR, Weinman SJ. Differential localization of annexins in ram germ cells: a biochemical and immunocytochemical study. J Histochem Cytochem 1991;39:955-63.

62. Palmerini CA, Carlini E, Nicolucci A, Arienti G. Increase of human spermatozoa intracellular $\mathrm{Ca} 2+$ concentration after fusion with prostasomes. Cell Calcium 1999;25:291-6.

63. Ho HC, Suarez SS. Characterization of the intracellular calcium store at the base of the sperm flagellum that regulates hyperactivated motility. Biol Reprod 2003;68:1590-6.

64. Jodar M, Kalko S, Castillo J, Ballescà JL, Oliva R. Differential RNAs in the sperm cells of asthenozoospermic patients. Hum Reprod 2012;27:1431-8.

65. Yamagata K, Murayama K, Okabe M, Toshimori K, Nakanishi T, Kashiwabara S, et al. Acrosin accelerates the dispersal of sperm acrosomal proteins during acrosome reaction. J Biol Chem 1998;273:10470-4. 